\title{
Non-symmetric 2-Designs Modulo 2
}

\author{
CHRIS M. SKINNER* \\ Department of Mathematics, University of Michigan, Ann Arbor, MI 48109
}

Communicated by D. Jungnickel

Received September 27, 1992; Revised June 9, 1992.

\begin{abstract}
Necessary conditions are obtained for the existence of a $2-(v, k, \lambda)$ design, for which the block intersection sizes $s_{1}, s_{2}, \ldots, s_{n}$ satisfy $s_{1} \equiv s_{2} \equiv \ldots \equiv s_{n} \equiv s\left(\bmod 2^{e}\right)$, where $e$ is odd. These conditions are obtained by combining restrictions on the Smith Normal Form of the incidence matrix of the design with some well known properties of self-orthogonal binary codes with all weights divisible by 4 .
\end{abstract}

\section{Introduction}

Let $\Theta$ be a $2-(v, k, \lambda)$ design where the block intersection sizes $s_{1}, s_{2}, \ldots, s_{n}$ satisfy $s_{1} \equiv s_{2} \equiv \ldots \equiv s_{n} \equiv s\left(\bmod p^{e}\right)$, where $p$ is a prime and $e$ is odd. Let $A$ be the incidence matrix of $\mathcal{B}$ and put

$$
X=\left[\begin{array}{c}
A \\
\lambda_{1} j
\end{array}\right] \text { and } X^{\prime}=\left[A^{t}-\lambda_{2} \dot{j}^{t}\right] \text {, }
$$

where $\lambda_{1}$ is a power of $p$ such that $\lambda_{1} \lambda_{2}=\lambda, \lambda_{1} \mid \lambda_{2}$ and $0 \leq\left(\lambda_{2}\right)_{p}-\left(\lambda_{1}\right)_{p} \leq 1$.

Using $X$ and $X^{\prime}$, one can construct a sequence of nested codes over ${ }_{F}$. Let $L$ and $L^{\prime}$ be the integral lattices spanned by the rows of $X$ and the columns of $X^{\prime}$, respectively. Let $\pi: \mathbb{Z}^{v} \rightarrow \mathbb{F}_{p}^{v}$ be the homomorphism that reduces every entry modulo $p$. For every integer $j \geq 0$, we define a code $X_{j}$ over $\mathbb{F}_{p}$ by

$$
X_{j}=\pi\left(p^{-j} L \cap \mathbb{Z}^{v}\right) \text {. }
$$

We define $X_{j}^{\prime}$ in like manner. In fact, given any $a \times b$ integer matrix $C$, we can construct a sequence of nested codes $C_{j}$ over $F_{p}$ in the same way as we did for $X$.

In [1] Blokhuis and Calderbank prove a number of properties of $X_{j}$ and $X_{j}^{\prime}$. Using these results they derive necessary conditions for the existence of a $2-(v, k, \lambda)$ design with all block intersection numbers congruent modulo $p^{e}$. However, the nature of these conditions are such that they are nontrivial only in the case where $p$ is odd. In this paper we combine the results of Blokhuis and Calderbank on $X_{j}$ and $X_{j}^{\prime}$ with known facts about selforthogonal binary codes to derive nontrivial necessary conditions in the case $p=2$. In particular, we prove the following theorem. 
TheOREM. Let $\mathbb{B}$ be a $2-(v, k, \lambda)$ design where the block intersection sizes $s_{1}, s_{2}, \ldots$, $s_{n}$ satisfy $s_{1} \equiv s_{2} \equiv \ldots \equiv s_{n} \equiv s\left(\bmod 2^{e}\right)$ and $e$ is odd. If $2^{e} \| r-\lambda$ then after possibly taking complements we have either

i) $v \equiv k+1 \equiv 0(\bmod 2)$ and $v \equiv 2(\bmod 8)$

ii) $v \equiv k \equiv 1(\bmod 4)$ and $v \equiv 1(\bmod 8)$

iii) $v \equiv k \equiv-1(\bmod 4)$ and $v \equiv-1(\bmod 8)$

iv) $v \equiv k \equiv 0(\bmod 2)$ and either $\left\{\begin{array}{l}(s)_{2} \equiv(v-k)_{2} \equiv 1(\bmod 2) \text { or } \\ v \equiv 2(\bmod 8)\end{array}\right.$

The proof of the theorem is in the same spirit as the corresponding results of [1] in the case $p$ odd. By extending $X$ or $X^{\prime}$ if necessary, a self-orthogonal code is constructed. However, instead of appealing to the intrinsic geometry of the code as in [1], we appeal to restrictions on the lengths of self-orthogonal codes to derive the existence conditions. This is an extension of the ideas in [2].

A similar application of self-dual codes to the existence of certain quasi-symmetric 2-designs can be found in [5].

\section{Proof of Theorem}

The proof of the theorem is broken into various cases, each of which is handled by one of the lemmas proved in this section. First, we set forth the results about binary codes that will be needed.

LEMMA 1 ([3]). The length of a self-dual binary code $C$ with all weights divisible by 4 is divisible by 8 .

LEMMA 2 ([4]). For $v$ odd, let $C$ be an $[v, 1 / 2(v-1)]$ self-orthogonal code such that all weights in $C$ are divisible by 4 . Then $v \equiv \pm 1(\bmod 8)$.

The next three lemmas provide all the results necessary to prove the theorem. The notation and terminology will be that of [1]. The 2-SNF (Smith Normal Form) of an integral matrix is the number $a_{i}$ of invariant factors $h$ for which $2^{i} \| h$. Fix $d=(e+1) / 2$.

Lemma 3. If $v \equiv k+1 \equiv 0(\bmod 2)$, then $v \equiv 2(\bmod 8)$.

Proof. In Lemma 5.2 of [1] it was proved that in this case $(\lambda)_{2}$ is odd, that $X$ and $X^{\prime}$ have different 2-SNFs, and that $\operatorname{dim} X_{d}^{\prime} \geq v / 2$. Rearranging the identity $\lambda(v-1)=r(k-1)$ gives $\lambda(v-k)=(r-\lambda)(k-1)$ which implies $(v-k)_{2} \equiv(k-1)_{2}(\bmod 2)$. Since $v-k$ is odd, we have $(k-1)_{2} \equiv 0(\bmod 2)$ and therefore $k \equiv 1(\bmod 4)$. The complementary design also has the property that $v$ is even and the block size $v-k$ is odd, so we deduce that $v-k \equiv 1(\bmod 4)$ and $v \equiv 2(\bmod 4)$. 
Let

$$
C=\left[\begin{array}{cc}
A & j^{t} \\
\lambda_{2} j & \lambda_{2}
\end{array}\right]
$$

and $D=\operatorname{diag}[1, \ldots, 1,-k]$. Recall that $s \equiv k\left(\bmod 2^{e}\right)$ and note that $k-1$ is even so $2^{e+1}|\lambda(v-k)| \lambda_{2}^{2}(v-k)$.

Let $z=\Sigma \mu_{i} r_{i}$ be a vector in the integer lattice $L$ spanned by the rows $r_{i}$ of $C$. Then the inner product with respect to $D$ of $z$ with itself is

$$
(z, z)=\sum_{i} \mu_{i}^{2}\left(r_{i}, r_{i}\right)+2 \sum_{i>j} \mu_{i} \mu_{j}\left(r_{i}, r_{j}\right)
$$

where

$$
\left(r_{i}, r_{i}\right)=\left\{\begin{array}{l}
k-k \\
\lambda_{2}^{2}(v-k)
\end{array} \equiv 0\left(\bmod 2^{e+1}\right)\right.
$$

and

$$
\left(r_{i}, r_{j}\right)=\left\{\begin{array}{l}
s-k \\
\lambda_{2}(s-k)
\end{array} \equiv 0\left(\bmod 2^{e}\right) .\right.
$$

It follows that $(z, z) \equiv 0\left(\bmod 2^{e+1}\right)$.

Next we prove that $C_{d}$ is self-orthogonal. If the binary vector $a \in C_{d}$, then there exists $z \in L$ such that $z \equiv 2^{d} a\left(\bmod 2^{d+1}\right)$. Thus $z=2^{d} a+2^{d+1} b$ and

$$
(z, z)=2^{2 d}(a, a)+2 \cdot 2^{2 d+1}(a, b)+2^{2 d+2}(b, b) \equiv 0\left(\bmod 2^{2 d+2}\right),
$$

so $(a, a) \equiv 0(\bmod 4)$.

Since $k \equiv 1(\bmod 4)$, the code

$$
C_{d}^{*}=\left\{\left(a_{1}, \ldots, a_{v-1}\right) \mid\left(a_{1}, \ldots, a_{v-1}, 0,0\right) \text { or }\left(a_{1}, \ldots, a_{v-1}, 1,1\right) \in C_{d}\right\}
$$

is self-orthogonal with all weights divisible by 4 . Since $\operatorname{dim} C_{d}^{*} \geq \operatorname{dim} X_{d}^{\prime}-1 \geq v / 2-1$, we have by Lemma 2 that $v-1 \equiv \pm 1(\bmod 8)$, and since $v \equiv 2(\bmod 4)$, we may conclude that $v \equiv 2(\bmod 8)$.

LeMma 4. If $v \equiv 1(\bmod 2)$ then after possibly taking complements either

(1) $k \equiv-1(\bmod 4)$ and $v \equiv-1(\bmod 8)$, or

(2) $k \equiv 1(\bmod 4)$ and $v \equiv 1(\bmod 8)$. 
Proof. After possibly taking complements we may suppose both $k$ and $v$ are odd. Let

$$
C=\left[\begin{array}{cc}
A & j^{t} \\
\lambda_{2} j & \lambda_{2}
\end{array}\right]
$$

and $D=\operatorname{diag}[1, \ldots, 1,-k]$. Note that $2^{e+1} \mid \lambda(v-k)$. Blokhuis and Calderbank prove that $X$ and $X^{\prime}$ have the same 2-SNF and that $\operatorname{dim} X_{d}^{\prime} \geq(v+1) / 2$. Note that $\operatorname{dim} C_{d} \geq$ $\operatorname{dim} X_{d}^{\prime} \geq(v+1) / 2$.

The argument employed in Lemma 3 proves that $(a, a) \equiv 0(\bmod 4)$ for all $a \in C_{d}$. If $k \equiv-1(\bmod 4)$ then the weight, $w t(a) \equiv(a, a) \equiv 0(\bmod 4)$. Thus $C_{d}$ is self-dual with all weights divisible by 4 , and so by Lemma $1 v \equiv-1(\bmod 8)$.

If $k \equiv 1(\bmod 4)$, let

$$
C_{d}^{*}=\left\{\left(a_{1}, \ldots, a_{v-1}\right) \mid\left(a_{1}, \ldots, a_{v-1}, 0,0\right) \text { or }\left(a_{1}, \ldots, a_{v-1}, 1,1\right) \in C_{d}\right\}
$$

Clearly $w t\left(a^{\prime}\right) \equiv 0(\bmod 4)$ for all $a^{\prime} \in C_{d}^{*}$. Since $\operatorname{dim} C_{d}^{*} \geq \operatorname{dim} C_{d}-1, C_{d}^{*}$ is self-dual with all weights divisible by 4 . Hence by Lemma 1 we have $v \equiv 1(\bmod 8)$.

LEMMA 5. If $v \equiv k \equiv 0(\bmod 2)$ then either

(1) $(s)_{2} \equiv(v-k)_{2} \equiv 1(\bmod 2)$, or

(2) $v \equiv 0(\bmod 8)$.

Proof. If (1) does not hold, then after taking complements if necessary, we may suppose $s=2^{2 n} \sigma$ where $\sigma$ is odd and $n \geq 1$. If $2 n>e$ then $X_{d}^{\prime}$ is a self-dual code with all weights divisible by 4 , and so $v \equiv 0(\bmod 8)$.

Therefore, suppose $2 n<e$. Let

$$
C=\left[\begin{array}{cc}
A & 2^{n} \boldsymbol{j}^{t} \\
\lambda_{2} \boldsymbol{j} & 2^{n} \lambda_{2}
\end{array}\right]
$$

and $D=\operatorname{diag}\left[1, \ldots, 1,-k / 2^{2 n}\right]$. By Lemma 5.2 of $[1](\lambda)_{2}$ is odd, $X$ and $X^{\prime}$ have different 2-SNFs, and $\operatorname{dim} X_{d}^{\prime} \geq v / 2$. Note $2^{e} \| \lambda(v-k)$, so that $(v-k)_{2}$ is even. Hence $v \equiv 0$ (mod 4). Since $(\lambda)_{2}$ is odd, we have $2^{e+1} \mid \lambda_{2}^{2}(v-k)$ and the arguments employed in Lemma 3 give $(a, a) \equiv 0(\bmod 4)$ for all $a \in C_{d}$.

If $k / 2^{2 n} \equiv-1(\bmod 4)$ then $(a, a) \equiv w t(a) \equiv 0(\bmod 4)$. It follows from Lemma 1 that $v+1 \equiv \pm 1(\bmod 8)$ and hence $v \equiv 0(\bmod 8)$. If $k / 2^{2 n} \equiv 1(\bmod 4)$ then let $C_{d}^{*}$ be as in Lemmas 3 and 4 . It follows that $v-1 \equiv \pm 1(\bmod 8)$ and we may conclude $v \equiv 0(\bmod 8)$.

The theorem is the result of combining Lemmas 3, 4 and 5 . 


\section{Conclusion}

We conclude this paper with a list of feasible parameter sets for which $2^{e} \| r-\lambda$, indicating those excluded by the conditions of the theorem (see Table 1). The following is a list for $s_{2}-s_{1}=8$ and $v<1500$. A minus $(-)$ indicates that the parameter set has been

Table 1. Possible parameter sets for 2-designs.

\begin{tabular}{|c|c|c|c|c|c|c|c|}
\hline$v$ & $k$ & $s_{1}$ & $s_{2}$ & $\lambda$ & $s$ & Case & Test \\
\hline 71 & 23 & 7 & 15 & 805 & 7 & $i i i$ & \\
\hline 93 & 45 & 21 & 29 & 690 & 5 & $i i$ & \\
\hline 130 & 52 & 20 & 28 & 172 & 4 & $i v$ & $*$ \\
\hline 161 & 65 & 25 & 33 & 520 & 1 & $i i$ & \\
\hline 210 & 70 & 22 & 30 & 418 & 6 & $i v$ & * \\
\hline 217 & 105 & 49 & 57 & 540 & 1 & $\ddot{i i}$ & \\
\hline 271 & 127 & 55 & 63 & 1905 & 7 & $i i i$ & \\
\hline 273 & 128 & 56 & 64 & 2176 & 0 & iic & \\
\hline 277 & 117 & 45 & 53 & 2691 & 5 & $i i$ & - \\
\hline 283 & 112 & 4 & 48 & 5264 & 0 & iiic & - \\
\hline 301 & 141 & 61 & 69 & 705 & 5 & $\ddot{i i}$ & - \\
\hline 302 & 106 & 34 & 42 & 4558 & 2 & $i v$ & - \\
\hline 309 & 144 & 64 & 72 & 672 & 0 & iiic & \\
\hline 325 & 117 & 37 & 45 & 810 & 5 & $i i$ & - \\
\hline 331 & 91 & 19 & 27 & 1001 & 3 & $i \dddot{i} i$ & - \\
\hline 331 & 155 & 67 & 75 & 465 & 3 & $i i i$ & - \\
\hline 337 & 112 & 32 & 40 & 896 & 0 & $\ddot{i} c$ & \\
\hline 341 & 165 & 77 & 85 & 510 & 5 & $i i$ & - \\
\hline 342 & 114 & 34 & 42 & 2046 & 2 & $i v$ & - \\
\hline 349 & 96 & 24 & 32 & 2784 & 0 & $i i c$ & - \\
\hline 351 & 111 & 31 & 39 & 3885 & 7 & $i i i$ & \\
\hline 371 & 35 & 3 & 11 & 185 & 3 & $i i i$ & - \\
\hline 378 & 108 & 28 & 36 & 1508 & 4 & $i v$ & $*$ \\
\hline 391 & 55 & 7 & 15 & 715 & 7 & $i i i$ & \\
\hline 475 & 75 & 11 & 19 & 237 & 3 & $i i i$ & \\
\hline 477 & 189 & 69 & 77 & 357 & 5 & $i i$ & - \\
\hline 495 & 144 & 40 & 48 & 304 & 0 & iiic & \\
\hline 511 & 175 & 55 & 63 & 425 & 7 & $i i i$ & \\
\hline 573 & 144 & 32 & 40 & 672 & 0 & $\ddot{i i c}$ & - \\
\hline 649 & 73 & 1 & 9 & 657 & 1 & $\ddot{i}$ & \\
\hline 651 & 155 & 35 & 43 & 325 & 3 & $i i i$ & - \\
\hline 657 & 81 & 9 & 17 & 738 & 1 & $\ddot{i} i$ & \\
\hline 715 & 187 & 43 & 51 & 357 & 3 & $i i i$ & - \\
\hline 770 & 110 & 14 & 22 & 1538 & 6 & $i v$ & $*$ \\
\hline 771 & 51 & 3 & 11 & 1309 & 3 & $i i i$ & - \\
\hline 801 & 81 & 1 & 9 & 720 & 1 & $\ddot{i i}$ & \\
\hline 806 & 140 & 20 & 28 & 3220 & 4 & $i v$ & $*$ \\
\hline 961 & 465 & 217 & 225 & 480 & 1 & $\ddot{i} i$ & \\
\hline 1066 & 246 & 54 & 62 & 426 & 6 & $i v$ & $*$ \\
\hline 1179 & 171 & 19 & 27 & 589 & 3 & $i i$ & - \\
\hline 1198 & 172 & 20 & 28 & 1204 & 4 & iv & $*$ \\
\hline 1353 & 105 & 1 & 9 & 910 & 1 & $i i$ & \\
\hline 1450 & 190 & 22 & 30 & 874 & 6 & iv & $*$ \\
\hline 1497 & 153 & 9 & 17 & 561 & 1 & $\ddot{i i}$ & \\
\hline
\end{tabular}


excluded by the theorem. An asterisk (*) indicates that though the theorem failed to eliminate this set, it is eliminated by results in [2]. We also indicate to which case of the theorem each parameter set corresponds, with a $c$ denoting a need to take the complement. All together, 26 of the 44 parameter sets are eliminated, 18 by the theorem.

Note that there exists a quasi-symmetric design with parameters $v=127, b=2007$, $r=651, k=31, \lambda=155$ with intersection numbers $x=7, y=15$; the blocks of this design are the 4-dimensional subspaces in $P G(6,2)$. These parameters do not appear in the table since $r-\lambda:=496$, which is divisible by $2^{e+1}=16$.

One notes that case $i$ ) of the theorem does not occur in the list. In fact, it can be shown that for $e=1$ this case is impossible (cf. [2]). It would be of interest to know whether or not this case can occur for $e>1$.

\section{Acknowledgment}

The author thanks A.R. Calderbank for suggesting this problem and for many helpful discussions. The author also thanks A. Blokhuis for generating the table of feasible parameter sets.

\section{References}

1. A. Blokhuis and A.R. Calderbank, Quasi-symmetric designs and the Smith Normal Form. Designs, Codes and Cryptography, Vol. 2, (1992), pp. 189-206.

2. A.R. Calderbank, The application of invariant theory to the existence of quasi-symmetric designs. J. Combinatorial Theory (A), Vol. 44, (1987), pp. 94-109.

3. A.M. Gleason, Weight polynomials of self-dual codes and the MacWilliams indentities, In Actes Conges Internl. de Mathématique, Vol. 3, pp. 211-315. Gauthier-Villars, Paris, (1971).

4. C.L. Mallows and N.J.A. Sloane, Weight enumerators of self-orthogonal codes, Discrete Math., Vol. 9, (1974), pp. $391-400$.

5. V. Tonchev, Quasi-symmetric designs and self-dual codes, European J. Comb., Vol. 7, (1986), pp. 67-73. 